\title{
Paediatric dentists' perception on a new local anesthetic drug Articaine in India-a cross sectional study
}

\author{
Abstract \\ Background: Articaine is a new local anaesthetic drug introduced to the Indian dental \\ market. \\ Objective: To elicit information regarding local anaesthetic selection, paediatric dentists' \\ perception on use of Articaine in children and factors which influence attitudes towards \\ acceptance of new technology.
}

Methods: A cross-sectional survey, that includes a 13 item questionnaire, was mailed to members of Indian Society of Pedodontics and Preventive Dentistry (ISPPD) with questions regarding details about Articaine use and scientific literature affect their adoption of new technology. Collected data was analysed descriptively.

Results: Of the sample, $46.7 \%$ responded, Lidocaine with epinephrine was the local anaesthetic preferred by most of the practitioners. Among the dentists' surveyed, $18 \%$ of them used Articaine, $36 \%$ informed that source of information regarding adoption of new technology was through colleagues whereas $23 \%$ reported that continuing professional development courses as their source.

Conclusion: Lidocaine with epinephrine was still the preferred local anaesthetic for use in children among Indian paediatric dentists. Use of Articaine became more prevalent as the child age increased.

Keywords: articaine, children, local anaesthetics, paediatric dentist, survey
Volume 9 Issue 4 - 2018

\author{
SVSG Nirmala, Naveen Kumar Kolli, Rupak \\ Kumar Dasararaju, Sivakumar Nuvvula \\ Department of Paedodontics \& Preventive Dentistry, Narayana \\ Dental College \& Hospital, India
}

\begin{abstract}
Correspondence: SVSG Nirmala, Professor, Department of Paedodontics\& Preventive Dentistry, Narayana Dental College \& Hospital, Nellore, Andhra Pradesh, India,
\end{abstract}

Email nimskrishna2007@gmail.com

Received: April I3, 2018| Published: July 05, 2018

\section{Introduction}

Like in other disciplines of science, Paediatric dentistry is a dynamic field, with constant advances in products and techniques. One such product is Articaine that has been recently introduced as a local aesthetic (LA) drug. During invasive dental procedures, prevention of pain can nurture the relationship of the child with dentist by building trust, allaying fear and anxiety and instill a positive dental attitude. ${ }^{1}$ In addition, while selecting local anaesthetic practitioners have to carefully consider the smaller size of children, who are more likely to develop toxic reactions from local aesthetics than adults because of their smaller anatomic proportions. ${ }^{2}$ By considering the above characteristics in children, it is paramount that the clinician chooses a local anaesthetic that will minimize the number of injections and volume of anaesthetic used while still allowing the required anaesthesia to be obtained. There are wide varieties of local anaesthetic agents that practitioners can use in children that satisfy the requirements for safe, effective local anesthesia in most operative cases. Two distinctive properties of Articaine related to its molecular structure make it as a desirable local anaesthetic for clinical use. First, Articaine is unique among amide local anaesthetics due to the presence of thiophene ring which makes it better lipid soluble. Due to its higher lipid solubility, it diffuses better through soft tissues than do other anaesthetics, thereby achieving higher intraneural concentration, more extensive longitudinal spreading, and better conduction blockade. Although much of the information is anecdotal, many dentists believe that this property of Articaine responsible for an increased success rate of local anesthesia. Second, Articaine gets metabolized both in plasma as well as tissue due to presence of an ester group, thus decreasing the risk of systemic toxicity. ${ }^{3}$ Articaine is a safe, well-tolerated and effective local anaesthetic for use in both children and adults. ${ }^{4}$ There is insufficient data regarding the response of Indian paediatric dentists advances in new technology with its adoption. This shows that there is lack of appropriate methods for introducing advances into dentistry. The purpose of this study was to determine:

1. Contemporary profile of Articaine users among paediatric dentists in India.

2. Their rational behind Articaine use or non-use.

3. The impact of different levels of education on their decisionmaking process.

\section{Materials and method}

A self-administered 13 item questionnaire was mailed to members of Indian Society of Pedodontics and Preventive Dentistry (ISPPD) in September 2016. A week later non-respondents were identified and questionnaire were mailed again. As a part of pilot study was sent to 5 paediatric dentists and 3 oral and maxillofacial surgeons and modified accordingly. Questions regarding sociodemographic data, perceptions on new technology, current local anesthetic use and Articaine use in children were included in the Questionnaire. Questions 11-13 focussed specifically on Articaine use, (Adverse effects caused by Articaine and perceived benefits of Articaine compared to lignocaine and reasons behind using and non-using Articaine). The project was approved by Narayana Dental College \& Hospital Ethical Committee. Data was compiled by using https://docs.google.com and descriptive analysis was done. 


\section{Results}

Of the 1120 paediatric dentists surveyed, 559 surveys were returned, yielding a gross response rate of $49.9 \%$. Of these 559 respondents 35 were excluded because of inappropriate responses so a sample of 524 (284 females, 240 males) surveys were analyzed; those overall response rate was $46.7 \%$. A majority of the survey population were females, practicing in urban area. Mean age of the participants was about 31.4 years; among them $76.5 \%$ is practicing exclusive paediatric dentistry (Table 1). Respondents were asked regarding knowledge about Articaine, one quarter of respondents knows completely about Articaine whereas an around 9\% of participant doesn't have knowledge about Articaine (Figure 1). Those respondents who mentioned use of Articaine stated that source of knowledge of Articaine was from their colleagues, CPD courses followed by company representatives an scientific journals (Figure 2). Respondents were asked to indicate which local anesthetic they preferred to use in paediatric population and the results are summarized in (Figure 3). Regarding the efficiency $42 \%$ reported that both were equally effective whereas $40 \%$ of respondents felt that Articaine was more efficacious than lignocaine (Figure 4). Most of the respondents were using Articaine for children above the age of 4 years $(84 \%)$. Respondents were asked to point out if any of their patients had experienced any adverse effects of local anesthesia that the respondent would attribute to Articaine. Among the Articaine users $66 \%$ of them reported no side effects, of $34 \%$ side effects reported by respondents, prolonged anesthesia $(45.1 \%)$ was most common side effect followed by transient paraesthesia $(24 \%)$. Among the Articaine users 55\% preferred to continue the usage. Main reason for not preferring Articaine in children was prolonged duration of anesthesia followed by economic concerns.

Table I Socio-demographic details

\begin{tabular}{ll}
\hline Gender & \\
Male & $240(45.8)$ \\
Female & $284(54.2)$ \\
Registered scope of practice & \\
General & $123(23.5)$ \\
Paediatric dentistry & $40 I(76.5)$ \\
Location of practice & \\
Rural & $82(15.3)$ \\
Urban & $442(84.3)$ \\
\hline
\end{tabular}

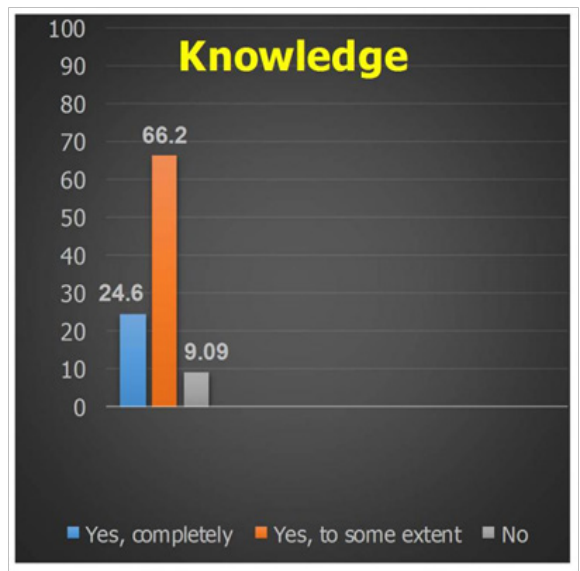

Figure I Knowledge about Articaine.

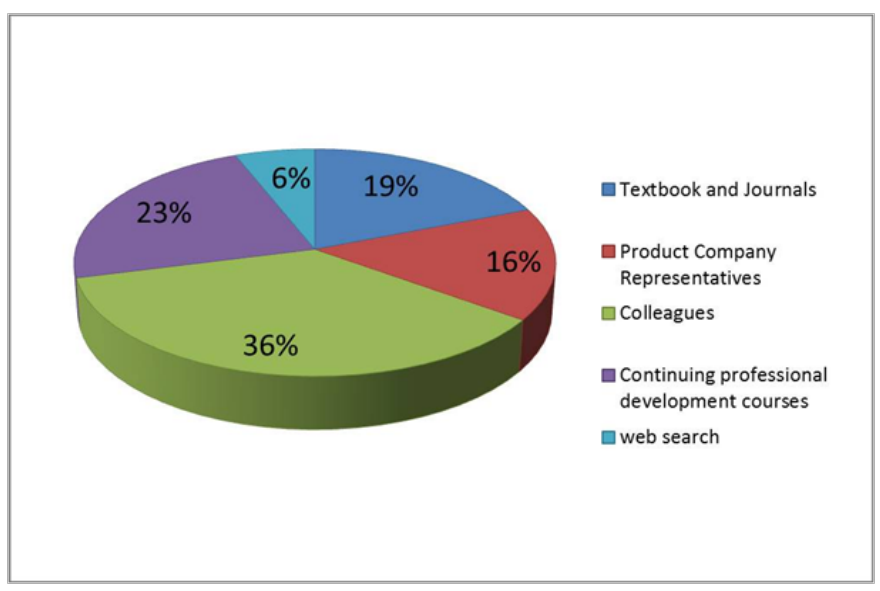

Figure 2 Methods of obtaining first information about Articaine.

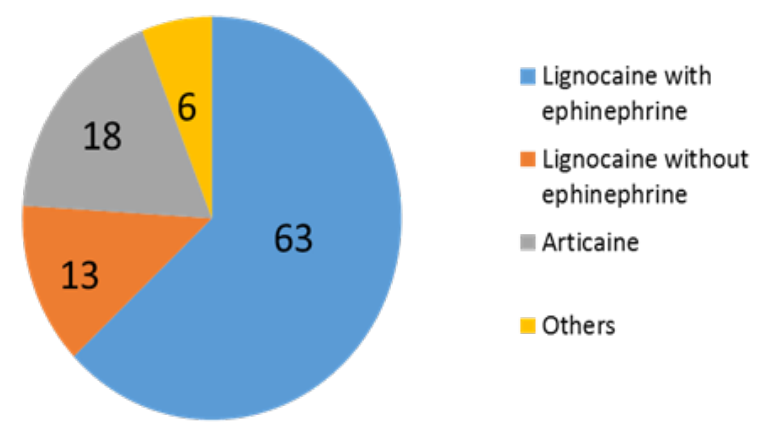

Figure 3 Paediatric dental practitioner preferred local aesthetic for use in children.

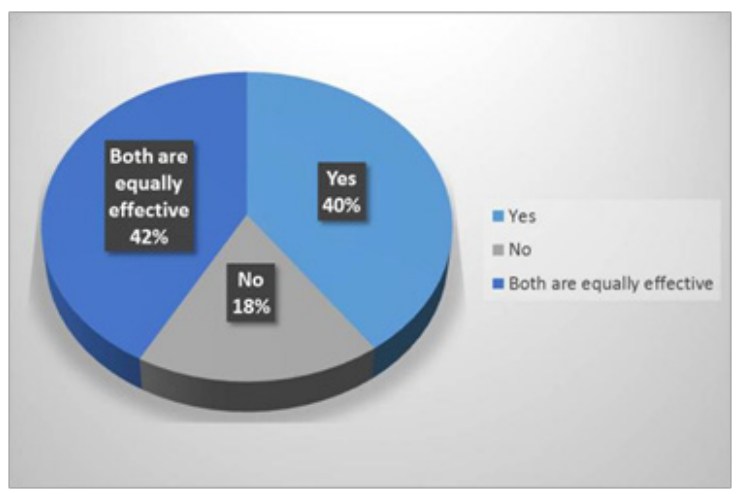

Figure 4 Regarding efficiency.

\section{Discussion}

The present study emphasises several factors regarding Indian paediatric dentist's perceptions of Articaine and also those that influence their decision making. The present study showed that Lidocaine with epinephrine is still the local anesthetic of choice among all the dentists surveyed and the results are similar to a study conducted by Brickhouse TH et al. ${ }^{5}$ Due to the introduction of Articaine into market there might be a small decrease in the preference of Lidocaine with epinephrine. Clinicians inaccessible to scientific literature and 
disinterest to seek contemporary literature were the reasons hindering them to adopt new technology. In this era of evidence-based dentistry, clinicians can make treatment decisions from the best available evidence. Continuing professional development courses are the best way to update about contemporary information and techniques for dentists. A recent systematic review suggests that multimethod and multiphased CPD has potential for the greatest impact on their clinical practices. ${ }^{6}$ Yet the literature shows mixed results; Articaine has comparable clinical properties to other available local anesthetics in several clinical settings with no demonstrable advantage whereas Articaine is more effective than lignocaine in providing successful anesthesia for routine dental procedures in the first molar region, ${ }^{7,8}$ this might be the reason for not preferring Articaine among the study population. Prolonged numbness and financial reasons influence use of Articaine. Maniglia-Ferreira $\mathrm{C}$ et al., ${ }^{9}$ reported that lignocaine was more economical compared to mepivacaine and Articaine. ${ }^{9}$ In a study by Adewumi A et al., ${ }^{10}$ prolonged anesthesia appears to be the most frequent adverse event observed in children younger than 7 years old. ${ }^{10}$ Present study results are contradictory to a study conducted among Australian dentists where Articaine was used by a majority of them. ${ }^{11}$ Limitations of this study are the survey comprised of limited selection of local anesthetics and the fact that many local anesthetic choices were included in the "others" selection. The type of treatment for which the practitioner was using Articaine has not been included.

\section{Conclusion}

Within the limitations of this study, it can be concluded that Lidocaine with epinephrine is the preferred local anesthetic preferred choice of local anesthetic in children. Majority of the respondents knows to some extent about Articaine, so appropriate education strategies has to be implemented, to update them with recent drugs and technology. And as the child age advances, there is an increased tendency for the use of Articaine.

\section{Acknowledgements}

None of the authors have any financial or personal interest in any of the products mentioned in this article.

\section{Conflict of interest}

The author declares that there is no conflict of interest.

\section{References}

1. American Academy of Pediatric Dentistry. Reference Manual 2007-08. Pediatr Dent. 2007;28:106-11.

2. Wright G, Weinberger S, Friedman $\mathrm{C}$, et al. The use of articaine local anesthesia in children under 4 years of age. Anesth Prog. 1989;36(6):268-7.

3. Oertel R, Rahn R, Kirch W. Clinical pharmacokinetics of articaine. Clin Pharmacokinet. 1997;33:417-25.

4. Malamed SF, Cagnon S, Leblanc D. A comparison between articaine HCL and lidocaine HCL in pediatric dental patients. Pediatr Dent. 2000;22(4):307-11.

5. Brickhouse TH, Unkel JH, Webb MD, et al. Articaine use in children among dental practitioners. Pediatr Dent. 2008;30:516-21.

6. Firmstone VR, Elley KM, Skrybant MT. Systematic review of the effectiveness of continuing dental professional development on learning, behavior, or patient outcomes. J Dent Educ. 2013;77:300-15.

7. Meechan J. Articaine and lignocaine. Evid Based Dent. 2011;12:21-2.

8. Brandt RG, Anderson PF, McDonald NJ, et al. The pulpal anesthetic efficacy of articaine versus lidocaine in dentistry: a meta-analysis. $J \mathrm{Am}$ Dent Assoc. 2011;142(5):493-504.

9. Maniglia-Ferreira C, Almeida-Gomes F, Carvalho-Sousa B, et al. Clinical evaluation of the use of three anesthetics in endodontics. Acta Odontol Latinoam. 2009;22(1):21-6.

10. Adewumi A, Hall M, Guelmann $M$, et al. The incidence of adverse reactions following $4 \%$ septocaine (articaine) in children. Pediatr Dent. 2008;30(5):424-8.

11. Yapp KE, Hopcraft MS, Parashos P. Dentists' perceptions of a new local anaesthetic drug--articaine. Aust Dent J. 2012;57(1):18-22. 